\title{
Shape Prior Model for Media-Adventitia Border Segmentation in IVUS using Graph Cut
}

\author{
Ehab Essa ${ }^{1}$, Xianghua Xie ${ }^{1}$, Igor Sazonov ${ }^{2}$, Perumal Nithiarasu ${ }^{2}$, and Dave \\ $\mathrm{Smith}^{3}$ \\ ${ }^{1}$ Department of Computer Science, Swansea University, UK \\ ${ }^{2}$ College of Engineering, Swansea University, UK \\ ${ }^{3}$ ABM University NHS Trust, Swansea, UK \\ \{csehab, x.xie, i.sazonov, p.nithiarasu\}@swansea.ac.uk
}

\begin{abstract}
We present a shape prior based graph cut method which does not require user initialisation. The shape prior is generalised from multiple training shapes, rather than using singular templates as priors. Weighted directed graph construction is used to impose geometrical and smooth constraints learned from priors. The proposed cost function is built upon combining selective feature extractors. A SVM classifier is used to determine an optimal combination of features in presence of calcification, fibrotic tissues, soft plaques, and metallic stent, each of which has its own characteristics in ultrasound images. Comparative analysis on manually labelled ground-truth shows superior performance of the proposed method compared to conventional graph cut methods.
\end{abstract}

Keywords: IVUS, graph cut, image segmentation, shape prior.

\section{Introduction}

Intra-vascular Ultrasound (IVUS) imaging is a catheter-based technology, which shows 2D cross-sectional images of the coronary artery. A typical IVUS image consists of lumen, vessel that includes intima and media layers, and adventitia that surrounds the vessel wall. The media-adventitia border represents the outer coronary arterial wall located between the media and adventitia. The media layer exhibits as a thin dark layer in ultrasound and has no distinctive feature. It is surrounded by fibrous connective tissues called adventitia. The appearance of the media-adventitia border in IVUS is affected by various forms of artifact, such as acoustic shadow which can be caused by catheter guide wire, dense fibrous tissue or calcification. Fig. 1 gives an example of IVUS image.

Segmentation in IVUS images has shown to be an intricate process and often requires user initialisation to achieve meaningful results. Among many others, graph based segmentation has shown to be a promising approach to IVUS segmentation. In [1], dynamic programming is used to search a minimum path in a cost function, which incorporates edge information with a simplistic prior based on echo pattern and border thickness. Manual initialisation is necessary. In [2], the authors applied spatio-temporal filters to enhance the lumen region based on 


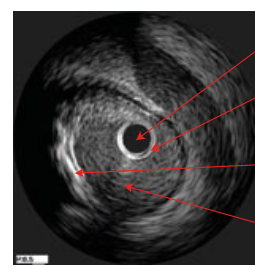

(a)

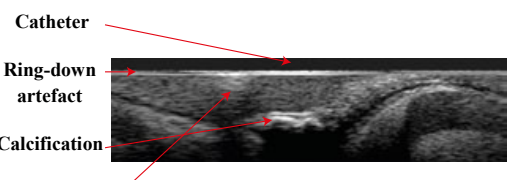

Guidewire artefact

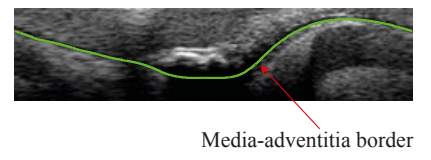

(c)

Fig. 1. Pre-Processing steps. (a) Original IVUS image. (b) Polar transformed image. (c) After removing the catheter region.

the assumption that the blood speckles have higher spatial and temporal variations than arterial wall. However, image features introduced by acoustic shadow or metallic stent would seriously undermine this assumption when searching for media-adventitia border. The $s$ - $t$ cut method [3] is employed in [4] to segment $3 \mathrm{D}$ IVUS data. Intensity distribution in the radial directions from catheter origin and regional features based on piecewise constant assumption are used to design the cost function. However, intensity based features are susceptible to artifacts.

Learning a priori using a set of representative shapes is an effective approach to impose a general constraint in searching global minimum using graph cut. Freedman and Zhang [5] defined the shape template as a distance function and embedded the average distance between every pair of pixels into the neighbourhood edges in the graph. However, this method effectively requires the user to place landmarks to define the initial shape. In [6], the authors proposed an iterative graph cut method. Kernel PCA was used to build the shape model. The method ignores the affine transformation, and needs a rectangle window initialisation of the location of the objects. Iterative graph cut framework was also adopted in [7]. The method penalises the terminal edges of the graph according to the similarity between the previous segmentation and the shape template.

In this paper, we propose an efficient graph cut algorithm to segment mediaadventitia border in IVUS images without user initialisation. Its objective functional consists of boundary based cost and shape penalties that are generalised from multiple training shapes. The boundary based features are dynamically selected to optimise the cost function based on trained classifier. The generalised shape prior is incorporated in the cost function, as well as embedded in graph construction. The method is evaluated on a large set of real data with groundtruth.

\section{Proposed Method}

The images are first transformed from Cartesian coordinates to polar coordinates and the catheter regions are removed (see Fig. 1). This transformation not only 
facilitates our feature extraction and classification but also transfers a closed contour segmentation to a "height-field" segmentation (see Fig. 1(c)). The border to be extracted intersects once and once only with each column of pixels. This particular form of segmentation allows us to construct a node-weighted directed graph, on which a minimum path can be found without any user initialisation.

\subsection{Graph construction without shape prior}

We first present our basic graph construction, following [8], which does not require user initialisation. Our extended version with incorporated shape prior will be discussed later in Section 2.5. Let $G=\langle V, E\rangle$ denote the graph, where each node $V(x, y)$ corresponds to a pixel in the transformed IVUS image $I(x, y)$ in polar coordinates. The graph $\mathrm{G}$ consists of two arc types: intra-column arcs and inter-column arcs. For intra-column, along each column every node $V(x, y)$, where $y>0$, has a directed arc to the node $V(x, y-1)$ with $+\infty$ weight assigned to the arc to ensure that the desired interface intersects with each column exactly once. In the case of inter-column, for each node $V(x, y)$ a directed arc with $+\infty$ weight is established to link with node $V\left(x+1, y-\Delta_{p, q}\right)$, where $\Delta_{p, q}$ is the maximum difference between two neighbouring columns $p$ and $q$ and acts as a smoothness constraint. Similarly, node $V(x+1, y)$ is connected to $V\left(x, y-\Delta_{p, q}\right)$. For IVUS segmentation, the first and the last columns are connected by intercolumn arcs to enforce connectivity. Finally, the nodes in the last row of the graph are connected to each other with $+\infty$ weight to maintain a closed graph. Inter-columns and intra-columns arcs are illustrated in Figure 2 (a).

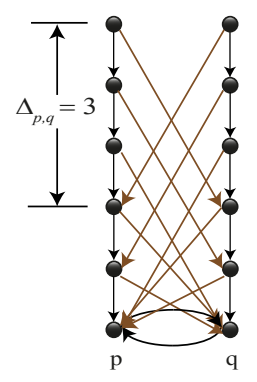

(a)

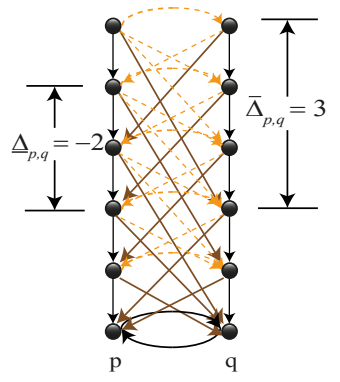

(b)

Fig. 2. Graph construction. (a) without shape prior where shape constraint is a global constant. (b) shape prior model (refer to Section 2.5 for details).

\subsection{Feature extraction and classification}

The media layer is usually thin and generally dark, and the adventitia layer tends to be brighter, see Fig. 1 as an example. Hence, edge based features are 
appropriate to extract the media-adventitia border. However, calcification and other interfering image features commonly exist above the media-adventitia border and cast acoustic shadows over the border, disrupting its continuity. Those imaging artifacts generally have large responses to image gradient based feature extraction. In this work, we propose to detect those artifacts and treat them differently when incorporating into the cost function.

To highlight the media-adventitia border, we use a combination of derivative of Gaussian (DoG) features and local phase features. A set of first and second order DoG filters are applied to capture the intensity difference between media and adventitia.

Local phase [9] has shown to be effective in suppressing speckles in ultrasound images. We use the dark symmetry feature [9] to highlight bar-like image patterns, which are useful to detect the thin media layer. This feature extraction operates at a coarser scale and complements to the edge features extracted using DoG filters.

For those parts of media-adventitia border that are beneath various forms of image artifacts, such as calcification, their image features are suppressed by those artifacts. Hence, it is desirable to detect those artifacts and treat those columns of pixels differently to others. However, instead of a usual attempt of localising those image artifacts based on intensity profile, e.g. $[10,11]$, which is problematic, we classify entire columns of pixels that contain those image artifacts. The detection result will then have an influence on the formulation of the cost function. To this end, we train a SVM classifier to classify individual columns of pixels in the polar coordinates into one of the following five categories: calcification, fibrous plaque, stent, guide-wire artifact, and normal tissue or soft plaque. Each of those has their characteristics; however, the difference between some categories may be small, e.g. calcification and fibrous plaque. To achieve efficient classification, the matching pursuit algorithm is used to reduce the number of support vectors.

\subsection{Boundary based cost function}

The boundary based energy term can be expressed as $E_{B}=\sum_{V \in S} \hat{c}_{B}(x, y)$, where $\hat{c}_{B}$ denotes the normalised cost function $\left(\hat{c}_{B}(x, y) \in[0,1]\right)$ and $S$ is a path in the directed graph. The formulation of the pre-normalisation cost function, $c_{B}$, is determined by the SVM classification result as presented below.

For normal tissue (or soft plaque), the media layer has a good contrast to adventitia. Hence, $c_{B}$ is defined as $c_{B}(x, y)=D_{1}(x, y)-D_{2}(x, y)$ where $D_{1}$ is a summation of raw filtering response of the first order DoG at four different orientations and $D_{2}$ denotes maximum response of second order DoG filtering from different orientations across three scales. That is $D_{1}$ measures total edge strength and $D_{2}$ is rotational invariant measurement of bar-like feature. Note, the media layer is generally darker than the lower layer, adventitia. The first order DoG filters are designed so that the stronger the media-adventitia border the lower the raw filtering response, i.e. negative values.

Calcified plaque exhibits strong edge features and casts varying degree of acoustic shadow on the media-adventitia border. Thus, we use the second or- 
der DoG responses to suppress calcification and enhance possible media layer. Fibrous tissue behaves similarly to calcification, except in majority cases mediaadventitia border is still discernible. Hence, bar feature detection is more appropriate and to enhance the effect we combine it with phase symmetry feature, i.e. $c_{B}(x, y)=-D_{2}(x, y)-F S(x, y)$ where $F S$ is the local phase feature.

The presence of stent causes scattering of ultrasound signals, leading to very bright pixels. Once stent is detected by SVM, it is straight forward to localise the stent region which should not be part of media or adventitia. The cost for stent region is assigned a positive constant. Second order DoG responses are used to assign cost value for non-stent region. As for guide-wire artifact, there are also very bright pixels but it casts complete shadow over entire column. Hence, we do not extract any feature and a positive constant is used as their cost value.

The cost for stent case is the second order DoG responses with more weight underneath the stent region. For guide-wire artefact, we do not extract any feature and a positive constant is used as their cost value, since it is a small area and cast acoustic shadowing.

\subsection{Shape prior based cost function}

The shape prior is defined as a likelihood term of each node in the graph, which is based on the similarity between the initial shape (obtained through finding the minimum closed set of our basic graph) and a set of templates from the training set. The graph construction is then modified so that inter-column arcs change dynamically according to the prior. The energy term for shape prior can be expressed as

$$
E_{S}=\sum_{x, y \in S} c_{P}(x, y)+\sum_{(p, q) \in \mathcal{N}} f_{p, q}(S(p)-S(q)),
$$

where $c_{P}$ denotes the cost function associated to prior and $f$ is a convex function penalising abrupt changes in $S$ between neighbouring columns $p$ and $q$ in the set $\mathcal{N}$ of neighbouring columns in the graph. The second term is realised through graph construction, detailed in the following Section 2.5. Notably in [7] the authors also used multiple templates in the graph cut. The terminal edge connection is determined by comparing the initial labelling with the template, e.g. if the node is in the template but not in the initial labelling, it connects to the source.

Each shape in the training set is treated as a binary template, $\psi$ where the area inside shape is one and the outside area is zero. The distance between two templates $\psi^{a}$ and $\psi^{b}$ is defined using a discrete version of Zhu and Chan distance [12]:

$$
d^{2}\left(\psi^{a}, \psi^{b}\right)=\sum_{P}\left(\psi^{a}-\psi^{b}\right)^{2} .
$$

where $P$ denotes the image domain. This distance measure is a true metric and is not influenced by image size. Affine transformation is also applied to remove the influence from geometrical transformation. Let $\Psi=\psi^{1}, \ldots, \psi^{N}$ denote the $N$ 
number of aligned shapes from the training set. Given a possible cut in the graph which produces an aligned binary shape $f$, its similarity to a shape template $\psi^{n}$ in the training set is computed as $\alpha\left(f, \psi^{n}\right)=\exp \left(-\frac{1}{2 \sigma^{2}} d^{2}\left(f, \psi^{n}\right)\right)$. Thus, the likelihood of this particular cut can be evaluated by taking into account of all training shapes:

$$
c_{R_{0}}=\frac{\sum_{n=1}^{N} \alpha\left(f, \psi^{n}\right) \psi^{n}}{\sum_{n=1}^{N} \alpha\left(f, \psi^{n}\right)} .
$$

In our case, an initial cut can be conveniently obtained by minimising the boundary based cost function alone. Note, it is fully automatic and there is no need for user initialisation. The labelling of the shape likelihood and initial cut needs to be compared in order to assign appropriate terminal arcs. The shape prior cost is defined as:

$$
c_{P}(x, y)=\lambda_{1}\left|c_{R_{0}}(x, y)-c_{R_{1}}(x, y)\right|,
$$

where $c_{R_{0}}$ and $c_{R_{1}}$ denote the cost associated to prior for the inferior region (the region under the border) and superior region (the region above the border) respectively, and $\lambda_{1}$ is the weight for the shape prior cost. The normalised weighted templates $c_{R_{0}}$ is in effect the inferior-region cost and is inversely proportional to the likelihood of a pixel belong to the region underneath the media-adventitia border. To define the superior-region prior $\operatorname{cost} c_{R_{1}}$, we simply compute the complement of $c_{R_{0}}$, i.e. $c_{R_{1}}=\max _{x, y} c_{R_{0}}(x, y)-c_{R_{0}}(x, y)$. As shown in Section 2.6, the shape prior cost $c_{P}(x, y)$ is used to assign weights for each pixel according to its position from the border. By assigning the shape prior cost in this way, we eliminate the need to identify the terminal connection type.

\subsection{Graph construction using shape prior}

In non-prior graph construction the inter-column maximum distance $\Delta$ is set as a constant. For our prior model, inter-column change should be influenced by the derived shape prior. In calculating the shape prior cost function, the training shapes are aligned to our initial graph cut. The inter-column changes are then generalised using mean $m_{p, q}$ and standard deviation $\sigma_{p, q}$ at individual column. These statistics are then used in determining maximum and minimum distances when connecting neighbouring columns in graph construction, i.e. $\bar{\Delta}_{p, q}=m_{p, q}+$ $c \cdot \sigma_{p, q}, \underline{\Delta}_{p, q}=m_{p, q}-c \cdot \sigma_{p, q}$, and $c$ is a real constant. Note, these inter-column arcs alone will impose a hard constraint on shape regularisation.

Hence, additional inter-column arcs are necessary in order to allow smooth transition (see dashed arcs in Fig. 2 (b)), that is intermediate values, $h \in$ $\left[\underline{\Delta}_{p, q}, \bar{\Delta}_{p, q}\right]$, are used to construct inter-column arcs. The direction of these arcs is based on the first order derivative of the function $f_{p, q}(h)$ as in (1). Here, we employ a quadratic function, $f_{p, q}=\lambda_{2}\left(x-m_{p, q}\right)^{2}$ where $\lambda_{2}$ is a weighting factor for smoothness constraint. If $f_{p, q}^{\prime}(h) \geq 0$ an arc from $V(x, y)$ to $V(x+1, y-h)$ is established; otherwise, the arc is connected from $V(x+1, y)$ to $V(x, y+h)$. The weight for these arcs is assigned as the second order derivative of $f_{p, q}$. Note, 
when $f_{p, q}^{\prime}(h)=0$, only single arc is defined to reduce the shape prior influence in presence of strong boundary features, instead of using bi-directional arcs on the mean difference $m_{p, q}$.

\subsection{Compute the minimum closed set}

The cost function $C(x, y)=c_{B}(x, y)+c_{P}(x, y)$ is inversely correlated to the likelihood that the border of interest passes through pixel $(x, y)$. The weight for each node on the directed graph can be assigned as:

$$
w(x, y)= \begin{cases}C(x, y) & \text { if } y=0 \\ C(x, y)-C(x, y-1) & \text { otherwise }\end{cases}
$$

For a feasible path $\mathcal{P}$ in the graph, the subset of nodes on or below $\mathcal{P}$ form a closed set and it can be shown that the cost of $\mathcal{P}$ is equivalent to the cost of nodes in the corresponding subset (differ by a constant) [8]. Hence, segmenting the media-adventitia is equivalent to finding the minimum closed set in the directed graph. The $s$ - $t$ cut algorithm [3] can then be used to find the minimum closed set, based on the fact that the weight can be used as the base for dividing the nodes into nonnegative and negative sets. The source $s$ is connected to each negative node and every nonnegative node is connected to the sink $t$, both through a directed arc that carries the absolute value of the cost node itself.

The smoothing parameter in graph construction prevents sudden drastic changes in the extracted interfaces. However, the segmented media-adventitia may still contain local oscillations. Here, efficient 1D RBF interpolation using thin plate base function is used to obtain the final interface.

\section{$3 \quad$ Experimental results}

A total of 1197 IVUS images of $240 \times 1507$ pixels in the polar coordinates from 4 sequences are used to evaluate the proposed method. These images contain various forms of fibrous plaque, calcification, stent, and acoustic shadow. Manual labelling was carried out on every 10 frames, i.e. 1197 frames in total, to establish groundtruth for quantitative analysis. The training set contains 278 images.

First, we compared our method against the $s-t$ cut algorithm [13]. The boundary cost function was kept the same, and careful manual initialisations were carried out for $s-t$ cut. The proposed method does not need user intervention. The first column in Fig. 3 shows typical results achieved using $s-t$ cut. Manual initialisations are shown in blue and green, and segmentation results are shown in red. Despite reasonable care in initialisation, the $s-t$ cut result was not satisfactory. The corresponding results of the proposed method are shown in the second column. The bottom of the each image shows the classification result of detecting different types of tissue. The proposed method achieved better accuracy and consistency. The quantitative comparison was carried out on a randomly selected subset of 50 images, since manual initialisation of $1197 \mathrm{im}-$ ages is too labour intensive. Table 1 shows that the proposed method clearly 
outperformed $s-t$ cut in both area difference measure (AD) and absolute mean difference measure (AMD) based on groundtruth.

Next, the proposed method was tested on the full dataset (1197 images) and its performance based on 1197 labelled groundtruth can be summarised as: $9.00 \%$ mean $\mathrm{AD}$ with standard deviation of 6.35 and 9.16 pixel mean AMD with standard deviation of 6.20 . This is marginally better than the first subset. Fig. 4 shows example comparisons to groundtruth. It is evident that the proposed method can handle various forms of ultrasound artifacts. Overall, the quantitative results suggest that the proposed method is an effective method in segmentation media-adventitia border in IVUS.

Table 1. Quantitative comparison to $s-t$ cut. AD: area difference in percentage; AMD: absolute mean difference in pixel in comparison to groundtruth.

\begin{tabular}{|c|c|c|c|c|}
\hline \multirow{2}{*}{} & \multicolumn{2}{|c|}{$s-t$ cut } & \multicolumn{2}{c|}{ proposed method } \\
\cline { 2 - 5 } & AD & AMD & AD & AMD \\
\hline Mean & 22.54 & 23.91 & 9.286 & 10.05 \\
\hline Std. & 8.87 & 7.49 & 5.03 & 5.41 \\
\hline
\end{tabular}
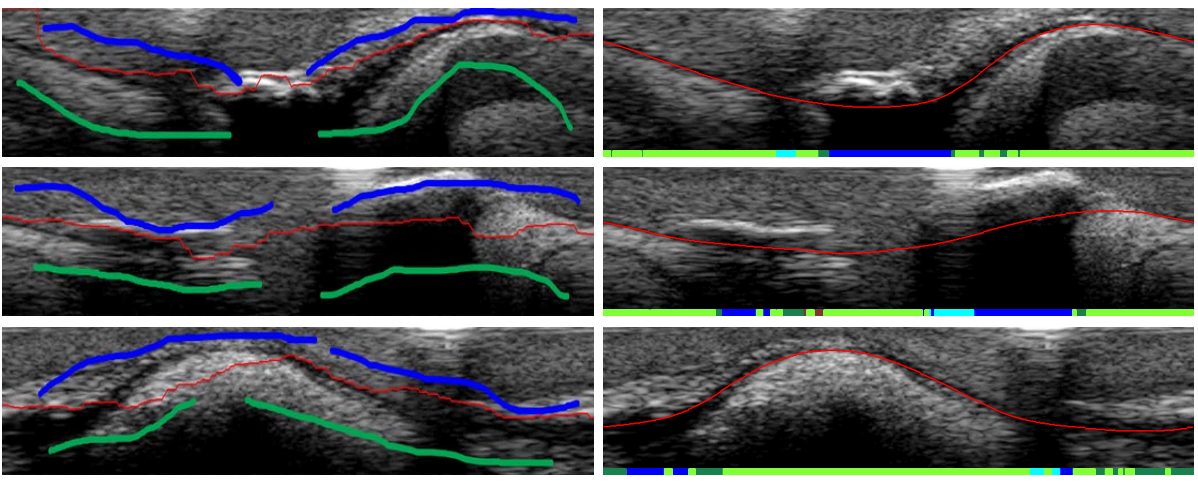

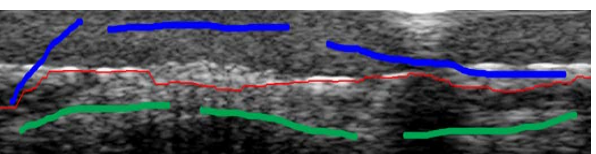

(a)

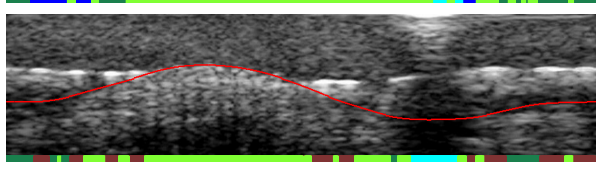

(b)

Fig. 3. (a) $s-t$ cut result (red) with user initialization (object: blue, background: green). (b) proposed method result; the bottom of each image also shows the classification result: calcified plaque (blue), fibrotic plaque (dark green), stent (dark red), guide-wire shadowing (cyan), and soft plaque/normal tissue (light green). 


\section{Conclusions}

We presented an automatic graph based segmentation method for delineating the media-adventitia border in IVUS images. Boundary based features were dynamically selected to optimise the cost function. The use of multiple training shapes proved to be beneficial. The generalised shape prior was used in both incorporating the cost function but also graph construction. Smoothness constraint was intrinsically imposed in graph construction. Qualitative and quantitative results on a large number of IVUS images showed superior performance of the method.

Acknowledgement - We would like to thank Welsh Government NISCHR for funding this research work (Grant ID: HA09/035).

\section{References}

1. Sonka, M., et al.: Segmentation of intravascular ultrasound images: A knowledgebased approach. T-MI 14 (1995) 719-732

2. Takagi, A., et al.: Automated contour detection for high frequency intravascular ultrasound imaging: A technique with blood noise reduction for edge enhancement. Ultrasound in Medicine and Biology 26(6) (2000) 1033-1041

3. Boykov, Y., Kolmogorov, V.: An experimental comparison of min-cut/max-flow algorithms for energy minimization in vision. T-PAMI 26(9) (2004) 1124-1137

4. Wahle, A., et al.: Plaque development, vessel curvature, and wall shear stress in coronary arteries assessed by x-ray angiography and intravascular ultrasound. MIA 10(1) (2006) 615-631

5. Freedman, D., Zhang, T.: Interactive graph cut based segmentation with shape priors. In: CVPR. (2005) 755-762

6. Malcolm, J., Rathi, Y., Tannenbaum, A.: Graph cut segmentation with nonlinear shape priors. In: ICIP. (2007) 365-368

7. Vu, N., Manjunath, B.S.: Shape prior segmentation of multiple objects with graph cuts. In: CVPR. (2008) 1-8

8. Li, K., Wu, X., Chen, D.Z., Sonka, M.: Optimal surface segmentation in volumetric images-a graph-theoretic approach. T-PAMI 28(1) (2006) 119-134

9. Mulet-Parada, M., Noble, J.: 2D + T acoustic boundary detection in echocardiography. MIA 4(1) (2000) 21-30

10. Filho, E., et al.: Detection \& quantification of calcifications in ivus by automatic thresholding. Ultrasound in Medicine and Biology 34(1) (2008) 160-165

11. Unal, G., et al.: Shape-driven segmentation of the arterial wall in intravascular ultrasound images. IEEE Trans. Info. Tech. in Biomed. 12(3) (2008) 335-347

12. Chan, T., Zhu, W.: Level set based shape prior segmentation. In: CVPR. (2005)

13. Boykov, Y., Funka-Lea, G.: Interactive graph cuts for optimal boundary and region segmentation of objects in n-d images. IJCV $\mathbf{7 0}(2)$ (2006) 109-131 

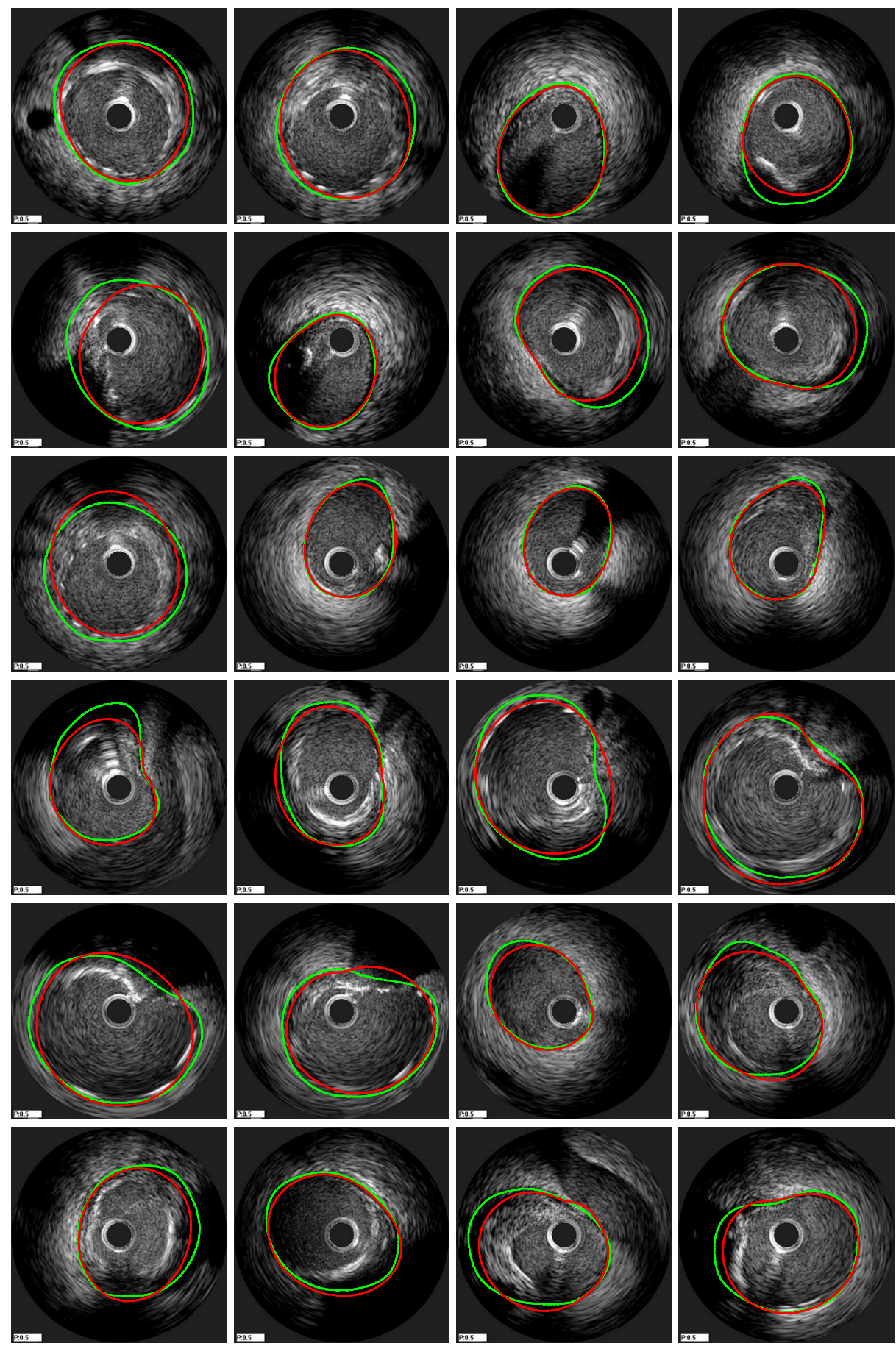

Fig. 4. Comparison between groundtruth (green) and the proposed method (red). 